\title{
La gestion de l'eau à l'aube du 3ème millénaire: Vers un paradigme scientifique nouveau [Tribune libre / Article bilingue] Water resources management at the turn of the millennium: towards a new scientific paradigm [Tribune libre] Water Resources Management at the Turn of the Millennium: Towards a New Scientific Paradigm [Free opinion]
}

\section{J. Ganoulis}

Volume 14, numéro 2, 2001

URI : https://id.erudit.org/iderudit/705418ar

DOI : https://doi.org/10.7202/705418ar

\section{Aller au sommaire du numéro}

\section{Éditeur(s)}

Université du Québec - INRS-Eau, Terre et Environnement (INRS-ETE)

\section{ISSN}

0992-7158 (imprimé)

1718-8598 (numérique)

\section{Découvrir la revue}

Citer ce document

Ganoulis, J. (2001). La gestion de l'eau à l'aube du 3ème millénaire: Vers un paradigme scientifique nouveau [Tribune libre / Article bilingue] Water resources management at the turn of the millennium: towards a new scientific paradigm [Tribune libre]. Revue des sciences de l'eau / Journal of Water Science, 14(2), 213-230. https://doi.org/10.7202/705418ar

\section{Résumé de l'article}

L'objectif de cette tribune est d'analyser la nouvelle approche concernant la gestion des ressources en eau qui a été adoptée par la communauté scientifique au seuil de ce nouveau millénaire. Après une révision de cette nouvelle approche, une méthodologie scientifique est proposée permettant d'exprimer le nouveau concept, qui est plutôt général et descriptif, en termes analytiques et quantitatifs, de façon qu'il soit appliqué dans des cas pratiques. Depuis quelques dizaines d'années déjà, il a été bien établi que la nouvelle approche va dans le sens de la gestion durable. Ceci veut dire qu'elle intègre des préoccupations sociales et environnementales aux critères traditionnels de performance technique et d'efficacité économique. La question qui se pose maintenant est comment le concept complexe de la durabilité, qui jusqu'à présent a été exprimé seulement de façon générale et qualitative, pourrait être formulé en termes analytiques et quantitatifs d'une méthodologie scientifique.

Sur le plan méthodologique, la modification des critères dont on doit tenir compte dans un cadre cohérent d'hypothèses et de raisonnements, suggèrent une évolution vers un paradigme scientifique nouveau. Le cadre général de ce paradigme que nous proposons est celui de l'analyse quantitative du risque à plusieurs dimensions.

Traditionnellement, l'objectif général de la gestion de l'eau, était la satisfaction de la demande dans diverses utilisations, comme l'agriculture, l'eau potable et l'industrie, en utilisant les ressources en eau disponibles de manière techniquement fiable et économiquement efficace. Dans cette approche, des solutions structuralistes et le plus souvent technocratiques, ont été proposées et réalisées dans plusieurs pays du monde. La construction de barrages et de réservoirs d'eau, la modification des lits des rivières et la dérivation des cours d'eau ont eu cependant, dans de nombreux cas, de sérieux impacts négatifs sur l'environnement et les conditions sociales. De plus, le gaspillage dans l'utilisation de cette ressource précieuse et la pollution galopante provenant de tous les secteurs d'utilisation de l'eau ont mis en question ce mode de gestion. Le concept de la gestion durable des ressources en eau a été évoqué, tout d'abord en 1972 à Stockholm, pendant la Conférence Mondiale des Nations Unies, puis à Rio, en 1992, avec l'Agenda 21. La nouvelle philosophie est basée sur la gestion intégrée de l'eau à l'échelle du bassin versant. Elle met l'accent sur la protection de l'environnement, la participation active des collectivités locales, la gestion de la demande, les aspects institutionnels, et le rôle de l'éducation continue tout le long de la vie de tous les utilisateurs d'eau.

Sur le plan méthodologique, la gestion intégrée de l'eau reste encore un problème ouvert où plusieurs approches cherchent à définir un paradigme cohérent. Dans cette tribune, nous en proposons un que nous appelons " le paradigme 4E " : Epistémique, Economique, Environnemental, Equitable. Il est basé sur l'analyse quantitative du risque à plusieurs dimensions : scientifique, économique, environnementale et sociale. Ce paradigme utilise soit la théorie des probabilités soit la logique du flou (ou les deux à la fois) afin d'évaluer et d'intégrer les risques technico - économiques et socio-environnementaux dans une perspective de gestion durable des ressources en eau.
Ce document est protégé par la loi sur le droit d'auteur. L’utilisation des services d’Érudit (y compris la reproduction) est assujettie à sa politique d'utilisation que vous pouvez consulter en ligne.

https://apropos.erudit.org/fr/usagers/politique-dutilisation/ 


\title{
La gestion de l'eau à l'aube du $3^{\mathrm{e}}$ millénaire : vers un paradigme scientifique nouveau
}

\author{
Water resources management at the turn \\ of the millennium: towards a new scientific paradigm
}

\section{J. GANOULIS ${ }^{1}$}

\section{RÉSUMÉ}

L'objectif de cette tribune est d'analyser la nouvelle approche concernant la gestion des ressources en eau qui a été adoptée par la communauté scientifíque au seuil de ce nouveau millénaire. Après une révision de cette nouvelle approche, une méthodologie scientifique est proposée permettant d'exprimer le nouveau concept, qui est plutôt général et descriptif, en termes analytiques et quantitatifs, de façon qu'il soit appliqué dans des cas pratiques.

Depuis quelques dizaines d'années déjà, il a été bien établi que la nouvelle approche va dans le sens de la gestion durable. Ceci veut dire qu'elle intègre des préoccupations sociales et environnementales aux critères traditionnels de performance technique et d'efficacité économique. La question qui se pose maintenant est comment le concept complexe de la durabilité, qui jusqu’à présent a été exprimé seulement de façon générale et qualitative, pourrait être formulé en termes analytiques et quantitatifs d'une méthodologie scientifique.

Sur le plan méthodologique, la modification des critères dont on doit tenir compte dans un cadre cohérent d'hypothèses et de raisonnements, suggèrent une évolution vers un paradigme scientifique nouveau. Le cadre général de ce paradigme que nous proposons est celui de l'analyse quantitative du risque à plusieurs dimensions.

Traditionnellement, l'objectif général de la gestion de l'eau, était la satisfaction de la demande dans diverses utilisations, comme l'agriculture, l'eau potable et l'industrie, en utilisant les ressources en eau disponibles de manière techniquement fiable et économiquement efficace. Dans cette approche, des solutions structuralistes et le plus souvent technocratiques, ont été proposées et réalisées dans plusieurs pays du monde. La construction de barrages et de réservoirs d'eau, la modification des lits des rivières et la déri-

†. Département de génie civil, Université Aristote de Thessaloniki, 54006 Thessaloniki, Grèce.

* Correspondance. E-mail :

** Les commentaires seront reçus jusqu'au 29 mars 2002. 
vation des cours d'eau ont eu cependant, dans de nombreux cas, de sérieux impacts négatifs sur l'environnement et les conditions sociales. De plus, le gaspillage dans l'utilisation de cette ressource précieuse et la pollution galopante provenant de tous les secteurs d'utilisation de l'eau ont mis en question ce mode de gestion. Le concept de la gestion durable des ressources en eau a été évoqué, tout d'abord en 1972 à Stockholm, pendant la Conférence mondiale des Nations Unies, puis à Rio, en 1992, avec l'Agenda 21.

La nouvelle philosophie est basée sur la gestion intégrée de l'eau à l'échelle du bassin versant. Elle met l'accent sur la protection de l'environnement, la participation active des collectivités locales, la gestion de la demande, les aspects institutionnels, et le rôle de l'éducation continue tout le long de la vie de tous les utilisateurs d'eau.

Sur le plan méthodologique, la gestion intégrée de l'eau reste encore un problème ouvert où plusieurs approches cherchent à définir un paradigme cohérent. Dans cette tribune, nous en proposons un que nous appelons « le paradigme 4E » : Épistémique, Économique, Environnemental, Equitable. Il est basé sur l'analyse quantitative du risque à plusieurs dimensions : scientifique, économique, environnementale et sociale. Ce paradigme utilise soit la théorie des probabilités soit la logique du flou (ou les deux à la fois) afin d'évaluer et d'intégrer les risques technico- économiques et socio-environnementaux dans une perspective de gestion durable des ressources en eau.

Mots clés : ressources en eau, gestion durable, analyse du risque.

\section{1 - INTRODUCTION : UN MONDE QUI CHANGE}

La gestion des ressources en eau en tant qu'activité scientifique et professionnelle, ne pouvait qu'être influencée par les changements socio-économiques que nos sociétés connaissent au seuil de ce nouveau millénaire. Dans l'histoire des sociétés, les changements de mode de production et de vie ne connaissent pas de discontinuité : ils sont plus ou moins rapides avec des périodes de plateau où les évolutions sont plus lentes suivies par des intervalles de temps pendant lesquels notre monde connait de profondes mutations. Telle est la période que nous vivons aujourd'hui avec la mondialisation de l'économie, l'accès à l'information, à la communication à distance et le progrès exponentiel de la science et de la technologie. Après la révolution agricole et industrielle, il est incontestable, qu'à l'aube du $3^{\mathrm{e}}$ millénaire, nous vivons la $3^{e}$ révolution, celle de l'information.

La gestion de l'eau, en tant que ressource naturelle n'a pu être exemptée de l'influence de ces changements socio-économiques. Au contraire, notre démarche scientifique et technique vis-à-vis de la gestion de cette ressource précieuse, a subi de telles modifications que nous pouvons affirmer qu'un nouveau paradigme de gestion a commencé à prendre forme. Ce paradigme est lié à notre nouvelle conception du monde, aux valeurs qui se sont établies et aux priorités accordées à ces valeurs.

Avant de préciser cette nouvelle conception, rappelons que la gestion de l'eau est une discipline horizontale, qui comporte plusieurs approches, comme l'approche: 
- scientifique : les sciences de l'eau et de la terre, comme l'hydrologie et l'hydrogéologie, les sciences de l'environnement, comme l'écologie et aussi les sciences fondamentales telles que la physique, la chimie et la biologie, étudient les propriétés et le comportement de l'eau en tant que ressource naturelle et aussi ses applications dans plusieurs domaines de l'activité humaine ;

- technique : l'hydrologie de l'ingénieur, l'hydraulique et d'autres disciplines en génie mécanique et chimique s'occupent de l'eau dans un contexte technologique ;

- économique : en considérant l'eau comme une ressource économique, le prix de l'eau et la tarification des services de l'eau peuvent être utilisés comme instruments de régularisation et d'utilisation rationnelle de l'eau ;

- juridique : la législation nationale, les directives et les normes et aussi des traités internationaux règlent les usages et la qualité des eaux à l'échelle nationale et internationale ;

- sociale : les sciences sociales mettent l'accent sur les attitudes socio-politiques, le comportement humain et la perception publique dans le domaine social de l'utilisation de l'eau.

En examinant les développements récents et les tendances des disciplines ci-dessus dans le domaine de l'utilisation et la gestion des ressources en eau, nous pouvons constater trois caractéristiques principales, résultant d'une influence certaine de la révolution informatique que nous vivons :

1. Contrairement à ce qui était admis il y a quelques dizaines d'années, il est maintenant généralement accepté que la science et la technologie seules ne peuvent résoudre les problèmes complexes de la gestion des ressources en eau.

2. L'importance de l'environnement et des retombées sociales des projets de gestion de l'eau ont été mises en évidence par plusieurs experts et organismes internationaux avec beaucoup de vigueur et de persuasion.

3. Les nouvelles techniques informatiques et de communication à distance ont révolutionné notre façon de travailler et ont modifié les moyens mis en œuvre pour les études de planification et de gestion des ressources en eau. Par exemple, l'emploi de Systèmes informatiques géographiques (SIG) en liaison avec des modèles mathématiques sophistiqués, la manipulation des bases de données digitalisées maintenant disponibles sur Internet et la mise en réseau des informations ont changé profondément notre approche scientifique et technique.

Ces changements ont entraîné la considération de la gestion des ressources en eau en tant que processus technico-social compliqué, où plusieurs disciplines doivent être prises en compte simultanément. La nouvelle approche intégrée a provoqué une modification des critères à prendre en compte et des méthodologies à utiliser de telle façon que nous pouvons affirmer qu'un nouveau paradigme scientifique dans le sens du T. KUHN (1962) est à définir.

Afin d'expliquer cette affirmation, le cadre traditionnel de la gestion des ressources en eau est tout d'abord rappelé. Les conséquences de ce mode de planification et de gestion ont montré que la protection de l'environnement et les conséquences sociales sont aussi importantes que la fiabilité technique et la performance économique. La crise actuelle de l'eau à l'échelle mondiale 
aussi bien sur le plan pratique que sur le plan méthodologique et institutionnel illustre bien les risques encourus et la nécessité d'un changement profond dans notre approche scientifique et technique.

Tel était le thème d'investigation du projet spécifique SP-E « Le paradigme européen de la gestion intégrée des ressources en eau " qui a été développé sous la coordination de l'auteur de cette tribune, dans le cadre de la première phase de Etnet - Environnement/eau. Etnet, qui continue à partir de l'an 2000 sous le nom d'Etnet21. C'est un réseau qui regroupe plus de 100 Universités et autres membres européens qui s'occupent de la gestion des ressources en eau (ETNET, 1998). La plupart des arguments et la base d'analyse de cette tribune ont bénéficié de l'expérience et des avis des collègues qui ont participé à l'élaboration du projet SP-E/Etnet.

\section{2 - L'APPROCHE TECHNICO-ÉCONOMIQUE : UN MODÈLE DE GESTION TRADITIONNEL}

L'approche traditionnelle de la gestion des ressources en eau met l'accent sur un objectif principal qui est la satisfaction des besoins de l'homme, aussi bien sur le plan de la quantité que sur le plan de la qualité de l'eau. Cette vision anthropocentrique est basée sur l'hypothèse implicite des quantités des ressources qui sont plus ou moins illimitées et que ce qui est bien pour satisfaire les besoins de l'homme est indépendant des conséquences sur l'environnement.

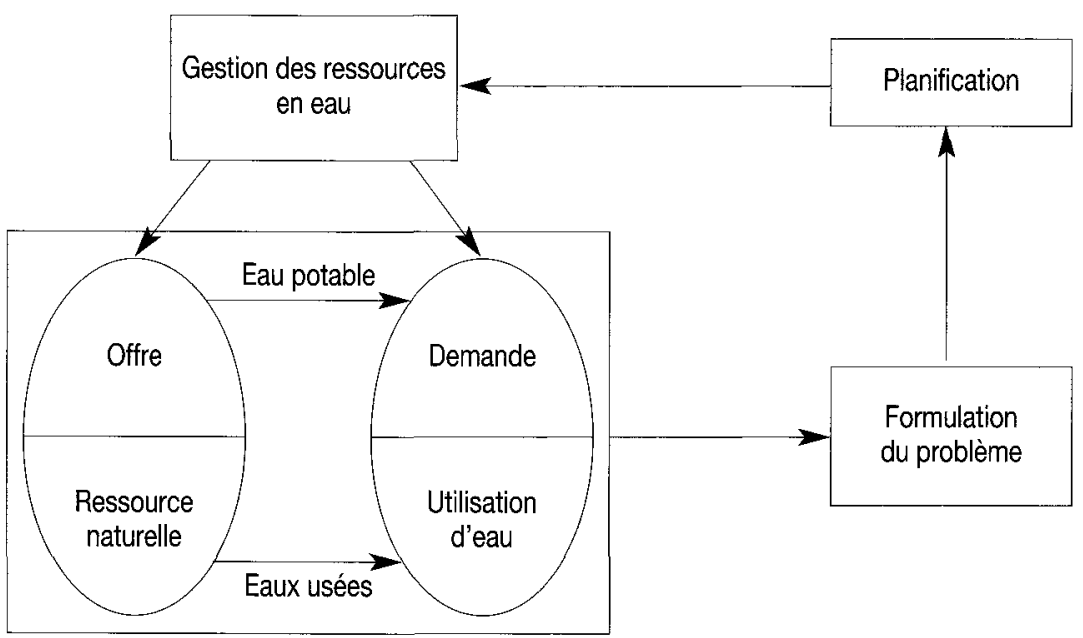

Figure 1 Gestion des ressources en eau potable dans les agglomérations.

Selon ce raisonnement, l'adduction de l'eau potable dans les agglomérations et l'amenée d'eau d'irrigation en agriculture et pour l'industrie, est basée sur une quantité de prélèvements d'eau qui doit satisfaire les besoins correspondants. Comme le montre schématiquement la figure 1, dans le cas de l'eau 
potable, le modèle de gestion met l'accent plutôt sur l'augmentation de l'offre en eau que sur la régulation de la demande ou les économies d'eau.

Si les quantités d'eau au voisinage de l'agglomération ne soient pas suffisantes, alors il faudrait faire des forages supplémentaires ou construire de longs aqueducs pour amener l'eau de loin, ou encore dévier des rivières avoisinantes. Suivant cette planification, suite à une concentration grandissante de la population et de la consommation par habitant, la ville d'Athènes s'alimente en eau potable située à une distance de plusieurs centaines de kilomètres, à l'ouest du pays.

Dans le cas de la protection contre les inondations, la solution traditionnelle est basée sur le principe ou bien de la rétention de la totalité du volume de la crue extrême loin de la zone à protéger ou sur l'évacuation rapide des flux d'eau le plus loin possible. Les solutions adoptées, que l'on pourrait qualifier de technocratiques, consistent soit à construire de grands barrages avec suffisamment de volume dans leur réservoir pour contenir le volume des crues, soit à modifier les lits des rivières en élargissant leur section, rectifiant les courbures et augmentant leur débit à l'aide du béton, souvent utilisé comme matériel de protection du lit à bas prix.

Les conséquences de ce mode de gestion ont été constatées dans plusieurs pays du monde et mis en évidence par plusieurs spécialistes et organismes internationaux. Tous les experts sont d'accord pour affirmer que le monde affronte actuellement une « crise de l'eau ». Ce n'est pas seulement une crise quantitative, bien que plusieurs pays soient à la limite de leurs ressources naturelles disponibles, mais c'est aussi une crise qualitative, due aux pollutions excessives et aussi une crise aux dimensions institutionnelle et sociale.

Le problème résultant de la mauvaise gestion des ressources d'eau est en partie dû à la politique sectorielle et au manque de coordination des organismes compétents aux niveaux national et international (GANOULIS et al., (eds.), 2000). II trouve des formes extrêmes dans certaines régions du monde. La destruction de la mer d'Aral due à la surexploitation des ressources en eau de la région, la disparition des zones humides en Europe et aux États-Unis, l'érosion des sols en Méditerranée et la pollution des grands fleuves sibériens en sont des exemples frappants.

\section{3 - VERS UNE GESTION DURABLE DES RESSOURCES EN EAU}

La crise de l'eau et les problèmes liés à l'environnement ont conduit à un changement drastique de notre attitude envers la gestion des ressources en eau.

Le terme de la gestion durable a remplacé celui de l'éco-développement, d'abord à la Conférence des Nations Unies en 1972 à Stockholm, puis repris et analysé au Sommet de Rio en juin 1992. Le concept de la gestion durable est évoqué dans la Déclaration de Rio, adopté à l'unanimité par 178 États, puis détaillé dans le Plan d'action 21, connu sous le nom de l'Agenda 21. Dans le principe 3 de cette déclaration, il est spécifié que la gestion durable « doit être 
réalisée de façon à satisfaire équitablement les besoins relatifs au développement et à l'environnement des générations présentes et futures ".

Cette affirmation est plutôt un souhait qu'une définition car elle ne décrit ni les moyens ni la démarche ni les actions à suivre afin d'arriver à réaliser ce souhait. Dans le domaine des ressources en eau, plusieurs organismes et associations ont essayé de définir les critères et la méthode à adopter afin de parvenir à une gestion durable (ASCE, 1998 ; ETNET, 1998 ; UNESCO, 1999). Dans un article récent, paru dans Water International, D.P. Loucks fait la synthèse sur les débats et diverses opinions concernant la gestion durable des ressources en eau (LOUCKS, 2000).

II en résulte que malgré le fait qu'une vision commune commence à se dessiner autour de la définition de la notion de durabilité, il n'y a pas encore un modèle simple et clair adopté par la communauté scientifique sur la méthodologie afin d'arriver à une planification durable de la gestion des ressources en eau.

II faut sans doute souligner que ce qui importe est l'analyse du pluralisme technologique. Ce qu'il faudrait est l'examen de tout le spectre des solutions technologiques possibles qui peuvent être situées entre deux extrêmes : les unes qui font appel aux techniques dites de pointe faisant appel aux nouvelles technologies et les autres étant simples ou traditionnelles et souvent à basse productivité. Parmi ces deux extrêmes, il faudrait pouvoir évaluer les impacts de ces solutions sur quatre dimensions:

1. la fiabilité scientifique et technique,

2. l'efficacité économique,

3. l'impact sur l'environnement, et,

4. l'équité sociale.

La solution durable devrait pouvoir gagner à la fois sur ces quatre critères et l'importance de l'un ou de l'autre pourrait être différente, selon les préférences locales. En utilisant la terminologie de la théorie des jeux, elle devrait être une solution à jeu de somme positive. En fait, comme le montre la Figure 2, la solution durable devrait minimiser les risques à quatre dimensions :

1) Scientifique/Technique, 2) Économique, 3) Environnementale et 4) Sociale.

Le paradigme scientifique que nous appelons 4E (EEEE : Épistémique, Économique, Environnemental et Équitable ; en Anglais : Epistemic, Economic, Environmental, Équitable) est basé sur l'analyse multidimensionnelle du risque et permet de tenir compte de ces quatre critères dans une perspective de gestion durable.

\section{4 - L'ANALYSE MULTICRITĖRE DU RISQUE}

L'idée principale de ce paradigme est que chaque alternative technique ou organisationnelle de gestion des ressources en eau comporte certaines possi- 
bilités de défaillance. Les conséquences de ces défaillances peuvent apparaître à plusieurs niveaux : technique, économique, environnemental et social. Elles peuvent être plus ou moins importantes selon le cas spécifique. Dans les cas extrêmes, les conséquences sont catastrophiques, comme par exemple sur le plan technique lors de la rupture d'un barrage ou, sur le plan économique, lors d'un crash bancaire pendant le financement d'un grand projet hydraulique.

En gestion des ressources en eau, les défaillances possibles, leur fréquence d'apparition et leurs conséquences sont calculables. Par exemple, le risque hydrologique est estimé lorsque le réseau des eaux pluviales déborde en ville à la suite d'une pluie de période supérieure à celle prévue par le projet. L'estimation de la fréquence de défaillance est précisément l'objet principal de la discipline scientifique connue sur le nom de l'analyse du risque. La quantification et la gestion du risque à plusieurs dimensions (technique, économique, environnementale et sociale) peuvent servir comme cadre général pour évaluer le degré de durabilité d'une stratégie alternative de gestion. (figure 2).

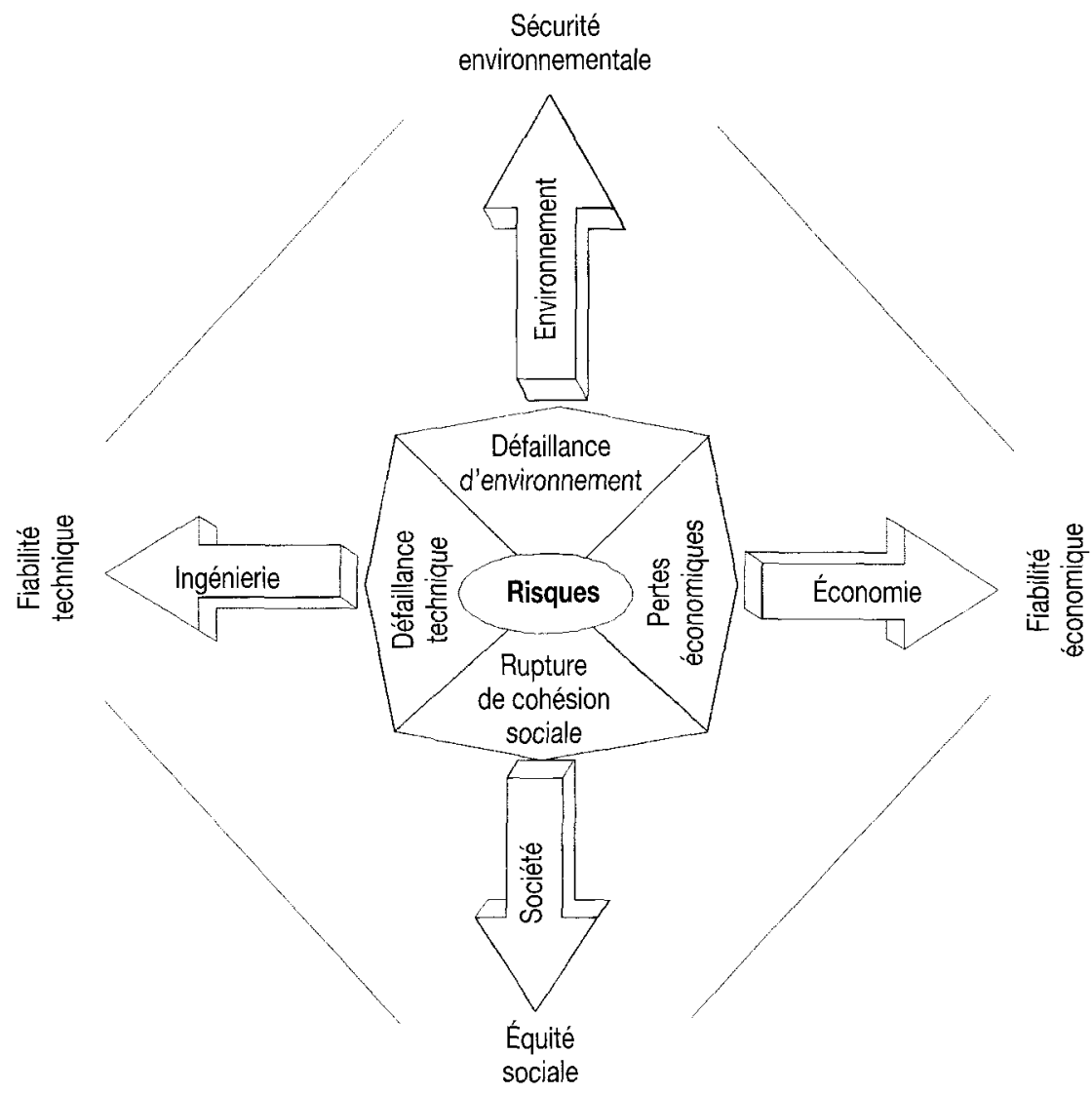

Figure 2 Risques et objectifs pour une gestion durable des ressources en eau. 
La théorie des probabilités ou la logique du flou (DUBOIS et PRADE, 1980) peuvent servir comme moyens d'évaluation des risques techniques, économiques, environnementaux et sociaux. Cette méthodologie a été appliquée dans le cas de la qualité de l'eau dans l'environnement (GANOULIS, 1994).

Bien que l'espace disponible de cette tribune ne soit pas suffisant pour décrire en détail la méthodologie proposée, nous pouvons énumérer les étapes principales :

1. Définir sur le plan conceptuel un ensemble de stratégies alternatives de gestion.

2. Estimer la matrice des risques technique, économique, environnemental et social correspondant à chaque stratégie alternative.

3. À l'aide d'un algorithme de prise de moyenne, calculer l'indice du risque composé technique et environnemental (risque technico-environnemental) et celui du risque composé social et économique (risque socio-économique).

4. Ranger les stratégies alternatives de gestion en utilisant comme critère dans l'espace bidimensionnel des risques composés, qui ont été définis précédemment (étape 3), la distance de chaque stratégie par rapport au point idéal (risque zéro).

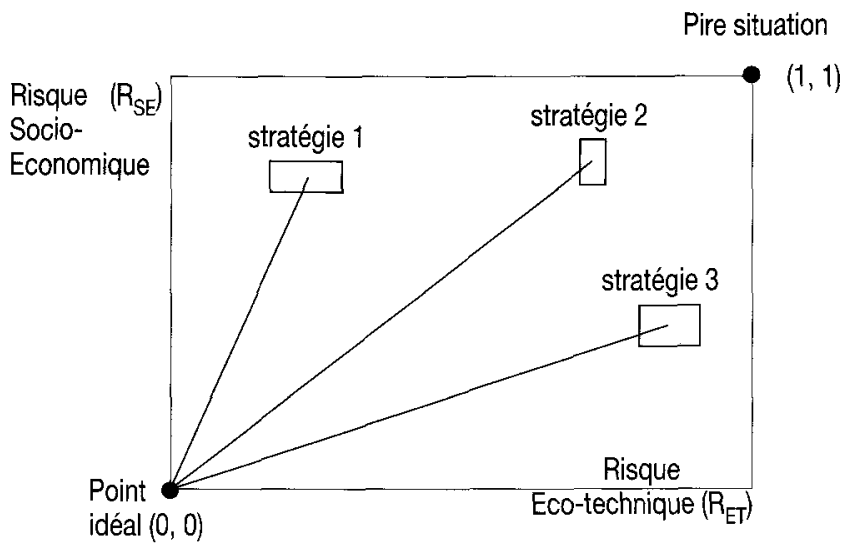

Figure 3 Classification des stratégies alternatives basée sur la distance par rapport au point idéal.

Cette méthodologie est liée à la théorie multicritère d'aide à la décision (GOICOECHEA et al., 1982 ; VINCKE, 1989 ; BOGARDI et NACHTNEBEL, 1994). Dans la figure 3 , les stratégies alternatives 1,2 et 3 sont représentées au plan bidimensionnel des risques composés par les coordonnées correspondant aux indices des risques technico-environnemental et socio-économique. Ces stratégies sont classées 1-3-2 en utilisant comme critère leurs distances par rapport au point idéal (risque zéro, solution durable idéale). 


\section{5 - CONCLUSIONS}

Les phénomènes liés aux ressources en eau présentent bien d'incertitudes, surtout à cause de leur variabilité dans le temps et dans l'espace. L'analyse du risque est une méthodologie appropriée afin d'estimer la fiabilité d'une solution de gestion de la ressource.

Parmi les stratégies alternatives, celle qui est durable devrait être à jeu de somme positive par rapport aux quatre indices de fiabilité : technique, environnemental, économique et sociale. Le paradigme $4 \mathrm{E}$ qui est décrit sommairement dans cette tribune permet d'estimer la solution la plus durable par une analyse multi-dimentionnelle du risque.

De plus, le risque doit être considéré comme fonction du temps, puisque, par définition, une solution durable doit préserver ses caractéristiques dans un proche et un plus lointain avenir.

\section{RÉFÉRENCES BIBLIOGRAPHIQUES}

ASCE, 1998. Sustainability Criteria for Water Resource Systems. ASCE, Reston, Virginia, USA, $253 \mathrm{p}$.

BOGARDI I., NACHTNEBEL H.P., 1994. Multicriteria Decision Analysis in Water Resources Management. IHP, UNESCO, Paris, $469 \mathrm{p}$.

DUBOIS D., PRADE H., 1980. Fuzzy Sets and Systems: Theory and Applications. Academic Press, New York.

ETNET Environment-Water, 1998. General Report for the Second Year of Activity. Van der Beken, VUB, Brussels.

GANOULIS J., 1994. Risk Analysis of Water Pollution: Probabilities and Fuzzy Sets. WILEY-VCH, Weinheim, Oxford, New York, $306 \mathrm{p}$.

GANOULIS J. et al. (eds), 2000. Transboundary Water Resources in the Balkans: Initiating a Sustainable Co-operative
Network. NATO ASI SERIES, Partnership Sub-Series 2, Environment, vol. 74, Kluwer Academic, Dordrecht, $254 \mathrm{p}$.

GOICOECHEA A., HANSEN D.R., DUCKSTEIN L., 1982. Multiobjective Decision Analysis with Engineering and Business Applications. J. Wiley, New York, $519 \mathrm{p}$.

KUHN T.S., 1962. The Structure of the Scientific Revolutions. The University of Chicago Press.

LOUCKS D.P., 2000. Sustainable Water Resources Management. Water International, vol. 25, $n^{\circ} 1,3-10$.

UNESCO, 1999. Sustainability Criteria for Water Resource Systems. Cambridge, U.K., Cambridge University Press.

VINCKE P., 1989. L'aide muiticritère a la décision. Éditions de l'Université de Bruxelles. 


\title{
Water resources management at the turn of the millennium: towards a new scientific paradigm
}

\author{
La gestion de l'eau à l'aube du $3^{\mathrm{e}}$ millénaire : \\ vers un paradigme scientifique nouveau
}

\section{J. GANOULIS ${ }^{1}$}

\section{SUMMARY}

The aim of this article is to analyze the new approach to water resources management adopted by the scientific community at the turn of the millennium. After reviewing the basic concept of this approach, a scientific methodology is proposed, in order to express the general and mostly descriptive new concept in analytical and quantitative terms, so that it may be applied in practical cases.

For several decades now the general concept of this new approach has been developing along the lines of sustainable development. This means that social and environmental considerations have been added to the traditional objectives of technical performance and economic effectiveness. The question now being raised is how the complex concept of sustainability, which until now has been expressed in general and descriptive terms only, may be formulated in the analytical and quantitative terms of a scientific methodology.

On the methodological level, the fact that several criteria and objectives within a coherent framework of hypotheses and reasoning are taken into account may suggest a move towards a new scientific paradigm. The general framework of the paradigm proposed in this paper is that of multidimensional quantitative risk analysis.

Traditionally, the general objective of water management has been the satisfaction of demand for various uses, such as agriculture, drinking water or industry, using available water resources in technically reliable and economically efficient ways. This approach has led to structural and mostly technocratic solutions being suggested and implemented in several countries. However in many cases, building dams, modifying riverbeds and diverting rivers has had serious negative repercussions on the environment and on social conditions. Moreover, waste in the use of this precious resource and

1. Department of Civil Engineering, Aristotle University of Thessaloniki, 54006 Thessaloniki, Greece.

* Correspondance. E-mail :

** Les commentaires seront reçus jusqu'au 29 mars 2002. 
rampant pollution in all areas of water use have raised doubts about this form of management. The concept of a sustainable management of water resources was first mentioned in Stockholm in 1972, during the United Nations World Conference and then at the Rio summit in 1992 with Agenda 21.

The new philosophy is based on the integrated management of water at the watershed basin level. Emphasis is placed on environmental protection, the active participation of local communities, the management of demand, institutional aspects and the role of continuous and lifelong education of all water users.

On the methodological level, integrated water management remains an open question and several different approaches are seeking to define a coherent paradigm. One possible paradigm is proposed in this article and may be called the " $4 \mathrm{E}$ paradigm": Epistemic, Economic, Environmental, Equitable. It is based on risk analysis, with a multidimensional character: Scientific, Economic, Environmental and Social. This paradigm uses either the theory of probability, or fuzzy logic, or both, in order to assess and integrate technicoeconomic and socio-environmental risks in a perspective of sustainable management of water resources.

Key words: water resources, sustainable management, risk analysis.

\section{1 - INTRODUCTION: A CHANGING WORLD}

As a scientific and professional activity, water resources management has inevitably been influenced by the socio-economic changes affecting society at the turn of the new millennium. History shows us that changes concerning production patterns and lifestyles are continuous, only the rate of change varies, with periods of slower evolution followed by periods of rapid and profound change. The world is currently going through a period of rapid change, with economic globalization, the development of information access and remote communication and the exponential progress of science and technology. After the agricultural and the industrial revolutions, there is no doubt that at the start of the $3^{\text {rd }}$ millennium, we are undergoing a $3^{\text {rd }}$ revolution, the information revolution.

The management of water as a natural resource could not fail to have been influenced by these socio-economic changes. Indeed, the former purely scientific and technical approach to the management of this precious resource has been modified to such a degree that it may be said that a new paradigm has started to emerge. This paradigm is related to our new view of the world, to the new values established and to the priorities granted to these values.

Before explaining this new view, it should be stated that water management is a horizontal discipline, where various approaches are possible:

- scientific: water and earth sciences, such as hydrology and hydrogeology, environmental sciences, such as ecology, as well as basic sciences like physics, chemistry and biology, study the properties and behavior of water as a natural resource as well as its applications in various fields of human activity. 
- technical: hydrological engineering, hydraulics and other disciplines of mechanical engineering deal with water in a technological context.

- economic: water in terms of being an economic resource, the price of water and the pricing of water services may be used as instruments for the regulation and rational use of water.

- legal: national legislations, directives and standards, as well as international treaties regulate water uses and quality at national and international levels.

- social: social sciences stress socio-political attitudes, human behavior and public perceptions regarding the use of water in a social context.

If we examine the recent developments and trends of the above disciplines in the field of water resources use and management, we can identify three main characteristics, which clearly result from the influence of the current revolution in information technology:

1. Contrary to the opinion generally prevailing a few decades ago, it is now widely admitted that science and technology alone cannot solve the complex problems of water resources management.

2. Several experts and international organisations have vigorously and persuasively demonstrated the importance of environmental consequences and social repercussions of water management projects.

3. New information technology (IT) and remote communication techniques have revolutionized working methods and modified the means used in planning and management studies of water resources. For example, the use of Geographic Information Systems (GIS) in conjunction with sophisticated mathematical models, the handling of digitized databases now available on the Internet and the networking of information have radically changed our scientific and technical approaches.

Due to these changes water resources management is now considered to be a complicated technico-social process involving several disciplines, and several different approaches need to be taken into account simultaneously. This new integrated approach has led to the criteria and methodologies used being modified to such an extent that one may claim that a new scientific paradigm, in the sense given to the word by KUHN (1962), needs to be specified.

In order to justify this claim, one must first recall what the traditional framework of water resources management was like. The consequences of traditional planning and management patterns have shown that environmental protection and social repercussions must be considered to be equally important as technical reliability and economic performance. The current worldwide water crisis on the practical, methodological and institutional levels is a clear illustration of the risks being run and of the need for a profound change in our scientific and technical approaches.

This was the research theme of the specific project SP-E entitled "the European Paradigm of Integrated Management of Water Resources," developed under the co-ordination of the author of this article, within the framework of the first phase of Etnet - Environment/water. Etnet, known since 2000 as Etnet21, is a network of more than 100 European universities and other institutions dealing with water resources management (ETNET, 1998). Most of the arguments as 
well as the basis of the analysis developed in this article have benefited from the experience and views of the colleagues who took part in the SP-E/Etnet project.

\section{2 - THE TECHNICO-ECONOMIC APPROACH: A TRADITIONAL MANAGEMENT MODEL}

The traditional approach of water resources management emphasizes one main goal: meeting human needs both in terms of water quality and quantity. This anthropocentric view is based on the implicit assumption that resources are unlimited, and that whatever helps satisfy human needs does not need to take environmental consequences into consideration.

According to this line of reasoning, the supply of drinking water in agglomerations and irrigation water for agriculture and industry is based on the available quantities of water. Figure 1 shows how in the case of drinking water, this management model emphasizes an increase in water supply.

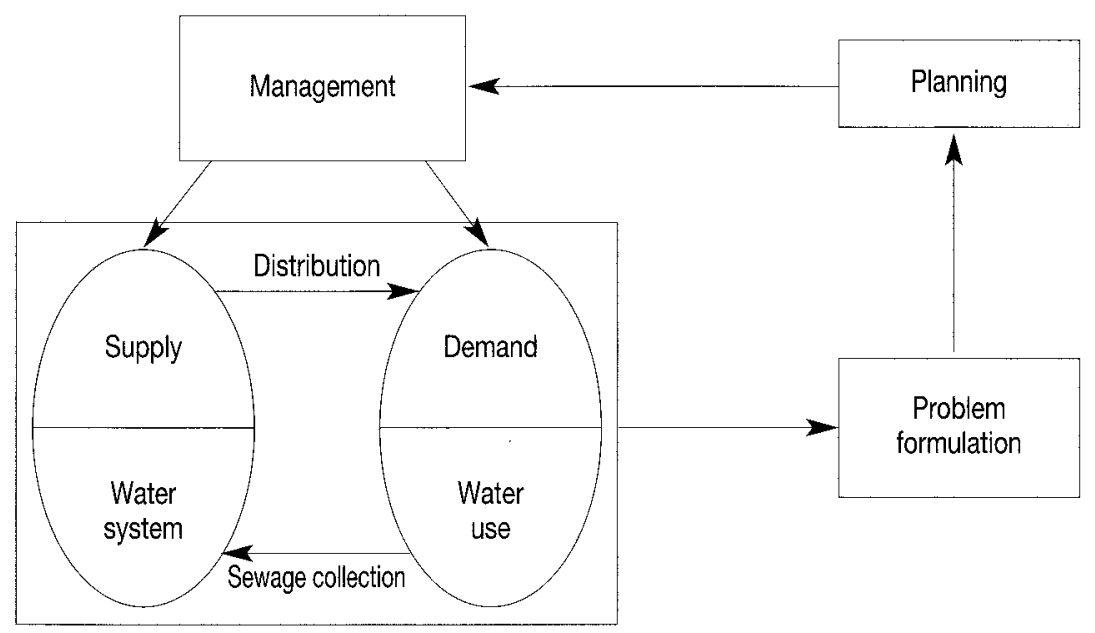

Figure 1 Water resources management in urban areas.

If nearby water supplies are inadequate, the additional drilling of wells, the building of long aqueducts to bring water from far away, or the diversion of nearby rivers is recommended. This type of planning has meant that for example in Athens, where there is an increase in urban population, drinking water is now brought from several hundred kilometers away.

In the case of flood protection, the traditional approach relies either on retaining the whole volume of the extreme rise in the water level away from the area to be protected, or on quickly redirecting the water as far away as possible. These technocratic solutions either meant building large dams with capa- 
cious reservoirs able to contain the flood volumes, or modifying riverbeds by widening their sections, straightening their reaches and increasing their flows by using concrete, chosen because of its low cost.

The consequences of this management pattern have been witnessed in several countries throughout the world and brought to light by several specialists and international organizations. Generally speaking, experts agree that the world is currently facing a "water crisis". This is not simply a quantitative crisis, even though several countries are reaching the limits of their resources, but also a qualitative crisis caused by pollution, all aspects having institutional and social repercussions.

Problems caused by faulty management of water resources are partly linked to a sectorial policy and the lack of co-ordination between responsible organizations on a national and international level (GANOULIS et al., 2000). These problems are acute in certain regions: for example the destruction of the Aral Sea caused by the overexploitation of the region's water resources, the disappearance of wetlands in Europe and the USA, ground erosion in the Mediterranean area and the pollution of large rivers in Siberia.

\section{3 - TOWARDS A SUSTAINABLE MANAGEMENT OF WATER RESOURCES}

The water crisis and environmental problems have led to a drastic change in attitudes towards water resources management.

The term "sustainable management" first replaced that of "eco-development" at the 1972 United Nations Conference in Stockholm and was then taken up and analyzed at the Rio summit in June 1992. The concept of sustainable management was mentioned in the Rio Declaration, unanimously adopted by 178 countries, and then detailed in Action Plan 21, now known as Agenda 21. In principle 3 of this Declaration, it is stated that sustainable management "must be implemented so as to meet in an equitable manner the needs related to development and to the environment of the present and future generations".

This statement is more of a wish than a definition, as it does not specify the means, the approach or the actions needed to achieve this objective. In the field of water resources, several organizations and associations have tried to define the criteria and the methodology to be used in order to achieve sustainable management (ASCE, 1998; ETNET, 1998; UNESCO, 1999). In a recent article published in Water International, D.P. Loucks reviews the debates and the various opinions concerning the sustainable management of water resources (LOUCKS, 2000).

This article shows that although a common definition of sustainability is starting to emerge, no simple and clear model has yet been defined and adopted by the scientific community. Such a model would concern the methodology to be used and the criteria to be taken into account, in order to achieve sustainable water resources management. 
It should perhaps be emphasized that what matters is the analysis of alternative technological solutions, where the whole spectrum of possible solutions should be studied. These solutions may lie between two extremes. Some of them rely on so-called advanced techniques using new technologies, while others are simple, traditional and often low productivity solutions. The impact of these solutions should be assessed in four areas:

1. Scientific and technical reliability

2. Economic effectiveness

3. Environmental impact

4. Social equity.

A sustainable solution should provide gains in all four of these areas, with priorities being established according to local needs. In game terminology, this would be known as a solution with a game of positive sums. As shown in figure 2, the sustainable solution should in fact minimize risks in four dimensions:

1) Scientific/Technical, 2) Economic, 3) Environmental and 4) Social.

The scientific paradigm called 4E (EEEE: Epistemic, Economic, Environmental, Equitable) is based on multidimensional risk analysis and allows the integration of these four criteria towards sustainable management.

\section{4 - MULTICRITERIA RISK ANALYSIS}

The main idea of this paradigm is that every technical or organizational alternative in water resources management involves a possibility of failure. The consequences of these failures may appear at several levels: technical, economic, environmental and social. How serious these failures may be will depend on each case. In extreme cases, the consequences are catastrophic, for example on the technical level when a dam bursts or, on the economic level when there is a bank crash or when finance is being sought for a large hydraulic project.

In the field of water resources management, possible failures, their frequency and their consequences can be calculated, as for example, the hydrologic risk of an urban rainwater network overflowing as a result of a period of rainfall greater than that provided for by a project. The evaluation of failure frequency is the main object of the discipline known as risk analysis. The quantification and management of risk on several dimensions (technical, economic, environmental and social) can be used as a general framework to evaluate the degree of sustainability of an alternative management strategy (figure 2).

The theory of probability or fuzzy logic (DUBOIS and PRADE, 1980) can be used as evaluation tools in technical, economic, environmental and social risk analysis. This methodology has been used to study water quality in the environment (GANOULIS, 1994). 


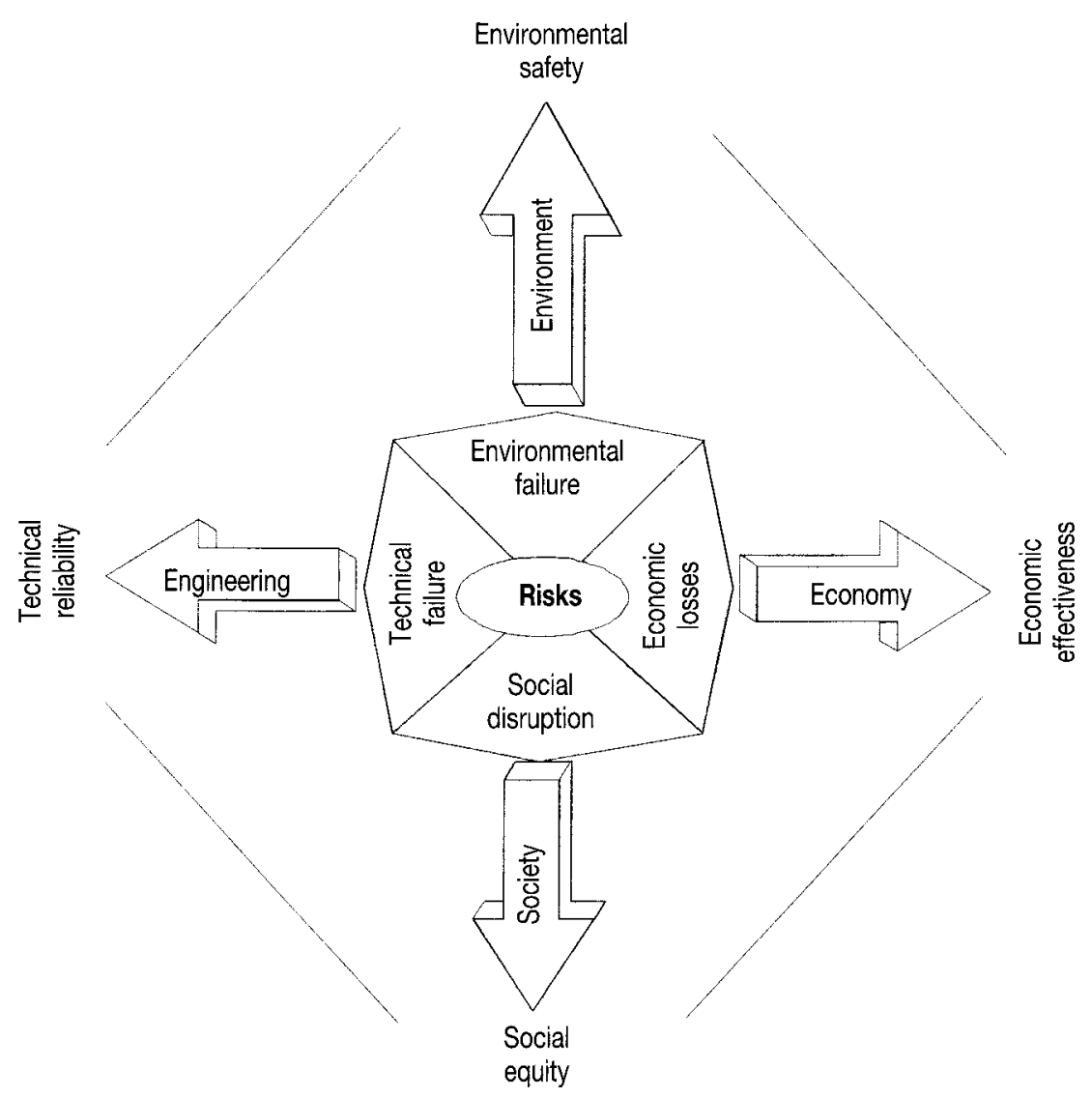

Figure 2 Risks and objectives for sustainable water resources management.

Briefly the main stages of the methodology are:

1. Establish a conceptual definition of a set of alternative management strategies.

2. Estimate the matrix of technical, economic, environmental and social risks corresponding to each alternative strategy.

3. Using an algorithm of average taking, calculate the index of technical and environmental composed risk (technico-environmental risk) and the index of social and economic composed risk (socio-economic risk).

4. Rank the alternative management techniques in a two-dimensional space along the criteria of the composed risks previously defined (stage 3) by estimating the distance to the ideal point ( 0 risk) for each strategy.

This methodology is linked to multicriteria decision analysis (GOICOECHEA et al., 1982; VINCKE, 1989; BOGARDI and NACHTNEBEL, 1994). It may be seen from figure 3 how alternative strategies 1,2 and 3 are represented in the two-dimensional space of composed risks by the co-ordinates corresponding to the technico-environmental and socio-economic risk indexes. These strategies are 


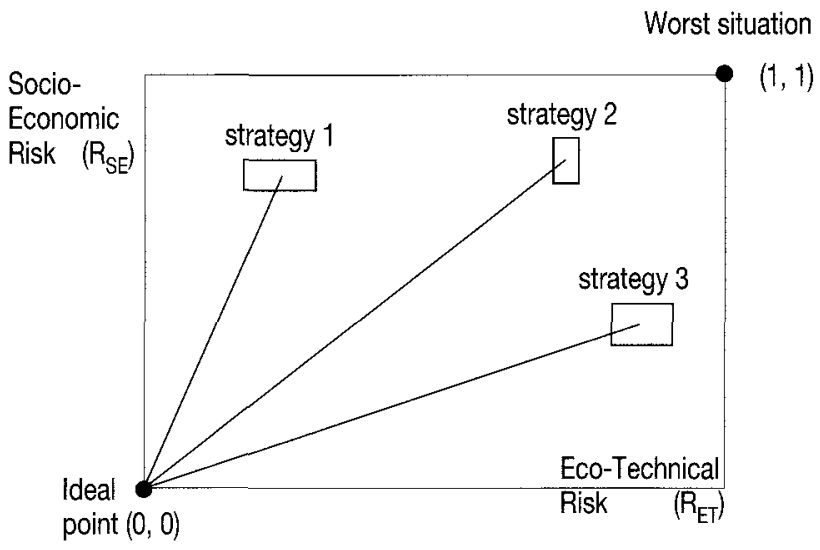

Figure 3 Distance-based ranking of alternative strategies.

ranked 1-3-2 according to their distance to the ideal point (zero risk, ideal sustainable solution).

\section{5 - CONCLUSIONS}

Phenomena related to water resources are characterized by a high degree of uncertainty, particularly because of their variability in time and space. Risk analysis is an appropriate methodology in order to assess the reliability of a resource management solution.

Among alternative solutions, the sustainable one should have a positive game sum on the four reliability indexes: technical, economic, environmental and social. The 4E paradigm, briefly described in this article, allows an assessment of the most sustainable solution using multidimensional risk analysis.

Moreover, the risk involved must be considered in relation to time, as a sustainable solution must, by definition, be one that maintains its characteristics in the near and more distant future.

\section{REFERENCES}

ASCE, 1998. Sustainability Criteria for Water Resource Systems. ASCE, Reston, Virginia, USA, $253 \mathrm{p}$.

BOGARDI I., NACHTNEBEL H.P., 1994. Multicriteria Decision Analysis in Water
Resources Management. IHP, UNESCO, Paris, $469 \mathrm{p}$.

DUBOIS D., PRADE H., 1980. Fuzzy Sets and Systems: Theory and Applications. Academic Press, New York. 
ETNET Environment-Water, 1998. General Report for the Second Year of Activity. Van der Beken, VUB, Brussels.

GANOULIS J., 1994. Risk Analysis of Water Pollution: Probabilities and Fuzzy Sets. WILEY-VCH, Weinheim, Oxford, NY, $306 \mathrm{p}$.

GANOULIS J. et al. (eds), 2000. Transboundary Water Resources in the Balkans: Initiating a Sustainable Co-operative Network. NATO ASI SERIES, Partnership Sub-Series 2, Environment, vol. 74, Kiuwer Academic, Dordrecht, 254 p.

GOICOECHEA A., HANSEN D.R., DUCKSTEIN L., 1982. Multiobjective Decision
Analysis with Engineering and Business Applications. J. Wiley, New York, 519 p.

KUHN T.S., 1962. The Structure of the Scientific Revolutions. The University of Chicago Press.

LOUCKS D.P., 2000. Sustainable Water Resources Management. Water International, 25(1), 3-10.

UNESCO, 1999. Sustainability Criteria for Water Resource Systems. Cambridge, U.K., Cambridge University Press.

VINCKE P., 1989. L'aide multicritère a la décision. Éditions de l'Université de Bruxelles. 